\title{
THE LICHNEROWICZ EQUATION IN THE CLOSED CASE OF THE EINSTEIN-MAXWELL THEORY
}

\author{
EMMANUEL HEBEY AND GIONA VERONELLI
}

ABStract. We investigate the existence of a solution and stability issues for the Einstein-scalar field Lichnerowicz equation in closed 3-manifolds in the framework of the Einstein-Maxwell theory. The results we obtain provide a complete picture for both the questions of existence and stability.

The fundamental equations of general relativity are the Einstein equations which link the curvature of the spacetime to its matter content. The equations, in the presence of a cosmological constant, are written as

$$
R_{\mu \nu}-\frac{1}{2} S g_{\mu \nu}+\Lambda g_{\mu \nu}=\frac{8 \pi G}{c^{4}} T_{\mu \nu},
$$

where $R_{\mu \nu}$ is the Ricci curvature tensor, $S$ is the scalar curvature, $g_{\mu \nu}$ is the Lorentzian metric tensor, $\Lambda$ is the cosmological constant, $G$ is Newton's gravitational constant, $c$ is the speed of light, and $T_{\mu \nu}$ is the stress-energy tensor. In the Einstein-Maxwell theory, $T_{\mu \nu}$ is the electromagnetic stress-energy tensor. Applying the conformal method of Lichnerowicz, and if we forget about the physical constant $8 \pi G / c^{4}$, solutions to the constraints equations are obtained by solving the system of unknowns $(u, W)$, a positive function and a vector field, consisting of the Lichnerowicz equation

$$
\Delta_{g} u+\frac{1}{8} S_{g} u=\frac{1}{4}\left(\Lambda-\frac{1}{3} \tau^{2}\right) u^{5}+\frac{|\sigma+D W|^{2}}{8 u^{7}}+\frac{|E|^{2}+|B|^{2}}{8 u^{3}}
$$

and the momentum constraint equation

$$
\operatorname{div}_{g}(D W)=\frac{2}{3} u^{6} \nabla \tau+E \times B,
$$

where $(M, g)$ is a smooth closed 3-dimensional Riemannian manifold, $S_{g}$ is the scalar curvature of $g, \sigma$ is a free 2-tensor field in $M, \tau$ is a free function in $M$ representing the mean curvature of the spacelike hypersurface $M, D$ is the conformal Killing operator, and $E, B$ are vector fields representing the electric and magnetic fields. When $\tau$ is constant, we see that the two equations (0.1) and (0.2) in the system are actually independent of one another. We assume here that $\tau$ is constant and consider that either $W=W(E, B)$ is given out of any physical considerations or that $W$ is the solution of (0.2). In the second case we need to assume that $(M, g)$ does not possess KIDs (this is somehow a generic situation; see Beig, Chrusciel and Schoen [3]) or that $E \times B$ is $L^{2}$-orthogonal to the subspace of conformal Killing fields. Then $W$ is $C^{1}$-controlled as soon as we control $E$ and $B$ in the $C^{0}$-topology (see Maxwell [15]). We let $\theta>0$ be constant and consider $\left(E_{\theta}, B_{\theta}\right)=\theta(E, B)$ so

Received by the editors October 26, 2011.

2010 Mathematics Subject Classification. Primary 58J05. 
that $\theta$ is the coupling constant which measures the strength of the interaction. To be coherent with (0.2), there should hold that $W\left(E_{\theta}, B_{\theta}\right)=\theta^{2} W(E, B)$. We let $W_{\theta}=\theta^{2} W(E, B)$, and for homogeneity reasons, we also let $\sigma_{\theta}=\theta^{2} \sigma$. We define

$$
K(\Lambda, \tau)=\Lambda-\frac{1}{3} \tau^{2}
$$

and let $Y(M, g)$ be the Yamabe invariant of $(M, g)$ given by

$$
Y(M, g)=\frac{1}{8} \inf _{\tilde{g} \in[g]} V_{\tilde{g}}^{-1 / 3} \int_{M} S_{\tilde{g}} d v_{\tilde{g}}
$$

where $[g]$ is the conformal class of $g$, and $V_{\tilde{g}}$ is the volume of $M$ with respect to $\tilde{g}$. We consider the Lichnerowicz equation

$$
\Delta_{g} u+\frac{1}{8} S_{g} u=\frac{1}{4} K(\Lambda, \tau) u^{5}+\frac{\left|\sigma_{\theta}+D W_{\theta}\right|^{2}}{8 u^{7}}+\frac{\left|E_{\theta}\right|^{2}+\left|B_{\theta}\right|^{2}}{8 u^{3}},
$$

and first ask the question of the existence of a solution to this equation. Existence results in the purely Einstein theory in closed manifolds go back to Isenberg [12. We also refer to Choquet-Bruhat, Isenberg and Pollack [5, Chrusciel, Galloway and Pollack 6], Hebey, Pacard and Pollack [11, and Maxwell [14]. Our answer to the question of existence in the case of (0.5) is as follows.

Theorem 0.1. Let $(M, g)$ be a smooth closed 3-manifold, $\Lambda, \tau$ be constants, $\sigma$ be a smooth $(2,0)$-tensor field, $(E, B) \not \equiv(0,0)$ be a nontrivial electromagnetic field, and $\theta>0$ be the coupling constant. Let $K(\Lambda, \tau)$ be as in (0.3) and $Y(M, g)$ be as in (0.4). Then,

(1) assuming $Y(M, g) \leq 0$, possesses a solution if and only if $K(\Lambda, \tau)<0$,

(2) assuming $Y(M, g)>0$ and $K(\Lambda, \tau) \leq 0$, (0.5) always possesses a solution,

(3) assuming $Y(M, g)>0$ and $K(\Lambda, \tau)>0$, there exists $\theta_{\star}>0$ such that (0.5) has a solution when $\theta<\theta_{\star}$, and no solution when $\theta>\theta_{\star}$.

As a remark, $\theta$ plays no role in (1) and (2) in the above theorem. It can be taken to be $\theta=1$. We assume from now on that $W=W(E, B)$ is controlled in the $C^{1}$-topology when $E$ and $B$ are controlled in the $C^{0}$-topology in the sense that for any $(E, B)$,

$$
W\left(E^{\prime}, B^{\prime}\right) \rightarrow W(E, B) \text { in } C^{1} \text { if }\left(E^{\prime}, B^{\prime}\right) \rightarrow(E, B) \text { in } C^{0} .
$$

As already mentioned, this is automatically the case if $W$ is obtained as a solution of the momentum equation (0.2). We define a perturbation of (0.1) to be any sequence of equations which we can write as

$$
\Delta_{g} u+\frac{1}{8} S_{g} u=\frac{1}{4} K\left(\Lambda_{\alpha}, \tau_{\alpha}\right) u^{5}+\frac{\left|\sigma_{\alpha}+D W_{\alpha}\right|^{2}}{8 u^{7}}+\frac{\left|E_{\alpha}\right|^{2}+\left|B_{\alpha}\right|^{2}}{8 u_{\alpha}^{3}}+k_{\alpha},
$$

where $W_{\alpha}=W\left(E_{\alpha}, B_{\alpha}\right),\left(\Lambda_{\alpha}\right)_{\alpha}$ and $\left(\tau_{\alpha}\right)_{\alpha}$ are sequences of real numbers, $\left(\sigma_{\alpha}\right)_{\alpha}$ is a sequence of 2-tensors, $\left(E_{\alpha}, B_{\alpha}\right)_{\alpha}$ is a sequence of electromagnetic fields, $\left(k_{\alpha}\right)_{\alpha}$ is a sequence of functions, and

$$
\begin{aligned}
& \Lambda_{\alpha} \rightarrow \Lambda \text { and } \tau_{\alpha} \rightarrow \tau \text { in } \mathbb{R}, \\
& \sigma_{\alpha} \rightarrow \sigma,\left(E_{\alpha}, B_{\alpha}\right) \rightarrow(E, B), \text { and } k_{\alpha} \rightarrow 0 \text { in } C^{0}
\end{aligned}
$$

as $\alpha \rightarrow+\infty$. Then, at least formally, (0.7) $\rightarrow$ (0.1) as $\alpha \rightarrow+\infty$. Following the terminology in Druet and Hebey $[\underline{8}$ we say that 
(i) (0.1) is bounded and stable if for any perturbation (0.7) of (0.1), and any sequence $\left(u_{\alpha}\right)_{\alpha}$ of solutions of (0.7), there exists a smooth positive solution of (0.1) such that, up to a subsequence, $u_{\alpha} \rightarrow u$ in $C^{1, \eta}$ for all $\eta \in(0,1)$,

(ii) (0.1) is stable if for any perturbation (0.7) of (0.1), and any bounded sequence $\left(u_{\alpha}\right)_{\alpha}$ in $H^{1}$ of solutions of (0.7), there exists a smooth positive solution of (0.1) such that, up to a subsequence, $u_{\alpha} \rightarrow u$ in $C^{1, \eta}$ for all $\eta \in(0,1)$.

Following standard terminology, (0.1) is said to be compact if for any sequence $\left(u_{\alpha}\right)_{\alpha}$ of solutions of (0.1), there holds that, up to a subsequence, $u_{\alpha} \rightarrow u$ in $C^{k}$ for all $k$ and some solution $u$ of (0.1). As one can check from elliptic theory, the $C^{1, \eta_{-}}$ convergences in (i) and (ii) improve to $C^{k, \eta}$-convergences when the convergences in (0.8) improve accordingly. There holds that bounded stability implies stability, and since (0.1) can clearly be seen as a perturbation of itself, bounded stability implies compactness as well. On the contrary, an equation can be compact and unstable (see Druet and Hebey [9]). Here we adopt the convention that an equation with no solution is stable (resp. bounded and stable) if it does not possess perturbations with $H^{1}$-bounded (resp. arbitrary) sequences of solutions. The theorem we prove concerning the stability of (0.1) is the following one.

Theorem 0.2. Let $(M, g)$ be a smooth closed 3-manifold, $\Lambda, \tau$ be constants, $\sigma$ be a smooth $(2,0)$-tensor field, $(E, B) \not \equiv(0,0)$ be a nontrivial electromagnetic field, and $W=W(E, B)$ satisfy (0.6). The Lichnerowicz equation (0.1) is bounded and stable if $K \neq 0$, and stable and compact if $K=0$, where $K=K(\Lambda, \tau)$ is given by (0.3).

When $K=0$, the equation cannot be bounded and stable without further assumptions, as we can check from the toy model $S_{g} \equiv 1, \sigma+D W \equiv 0,|E|^{2}+|B|^{2}=1$. Then

$$
\Delta_{g} u_{\alpha}+\frac{1}{8} u_{\alpha}=\frac{1}{4} K_{\alpha} u_{\alpha}^{5}+\frac{1}{8 u_{\alpha}^{3}}
$$

for $u_{\alpha} \equiv \alpha$, where $K_{\alpha}=\frac{1}{2 \alpha^{5}}\left(\alpha-\frac{1}{\alpha^{3}}\right)$. In particular, $K_{\alpha} \rightarrow 0$ as $\alpha \rightarrow+\infty$, while, obviously, in this example, $\left\|u_{\alpha}\right\|_{L^{p}} \rightarrow+\infty$ as $\alpha \rightarrow+\infty$ for any $1 \leq p \leq+\infty$. This contradicts bounded stability.

Some consequences of Theorem 0.2 are as follows. As a first consequence we assume (0.6). Then it easily follows from Theorem 0.2 that the solution in point (3) of Theorem 0.1 exists as well when $\theta=\theta_{\star}$. In other words, assuming (0.6), $Y(M, g)>0$, and $K(\Lambda, \tau)>0$, we get that there exists $\theta_{\star}>0$ such that (0.5) has a solution when $\theta \leq \theta_{\star}$, and no solution when $\theta>\theta_{\star}$.

As another consequence, if we still assume (0.6), that $Y(M, g)>0$, and that $K(\Lambda, \tau)>0$, then we get from Theorem 0.2 that we can actually compute the degree of our equation thanks to the existence and nonexistence parts in Theorem 0.1. More precisely, we know from Theorem 0.1 that (0.5) has a solution when $\theta>0$ is small, and no solution when $\theta$ is large, while we have by Theorem 0.2 that for any $\theta_{1}<\theta_{2}$, the family of equations (0.5) inherits compactness for $\theta \in\left[\theta_{1}, \theta_{2}\right]$. Given $A>0$ and $\eta \in(0,1)$, we define

$$
\Omega_{A}=\left\{u \in C^{2, \eta} \text { s.t. }\|u\|_{C^{2, \eta}}<A \text { and } \min _{M} u>\frac{1}{A}\right\} .
$$

We fix $\theta_{1}<1<\theta_{2}$ such that (0.5) has a solution when $\theta=\theta_{1}$ and no solution when $\theta=\theta_{2}$, and define $F_{\theta}: \overline{\Omega_{A}} \rightarrow C^{2, \eta}$ by

$$
F_{\theta}(u)=u-\mathcal{L}^{-1}\left(\frac{1}{4} K(\Lambda, \tau) u^{5}+\frac{\left|\sigma_{\theta}+D W_{\theta}\right|^{2}}{8 u^{7}}+\frac{\left|E_{\theta}\right|^{2}+\left|B_{\theta}\right|^{2}}{8 u^{3}}\right),
$$


where $\mathcal{L}(u)=\Delta_{g} u+\frac{1}{8} S_{g} u$. By the compactness of the family of equations (0.5) when $\theta \in\left[\theta_{1}, \theta_{2}\right]$, we get that there exists $A_{0} \gg 1$ such that $F_{\theta}^{-1}(0) \subset \Omega_{A}$ for all $\theta \in\left[\theta_{1}, \theta_{2}\right]$ and all $A \geq A_{0}$. Then we can define the Leray-Schauder degree $\operatorname{deg}\left(F_{\theta}, \Omega_{A}, 0\right)$, and by homotopy invariance, since equation (0.5) has no solution when $\theta=\theta_{2}$, we get that

$$
\operatorname{deg}\left(F_{\theta}, \Omega_{A}, 0\right)=0
$$

for all $A \gg 1$. In particular, generically speaking, it follows from $(\underline{0.9})$ that solutions of (0.5) come by pairs. A similar remark was carried out by Schoen 18, for the Yamabe equation whose degree, as computed in [18], turns out to be -1 .

It can be noted that (0.1) arises in different Einstein-matter field theories as in the Einstein perfect fluids theory. We refer to Isenberg, Maxwell and Pollack 13. for more details on the building of these equations. Multiplicity for (0.1) when $(E, B) \equiv(0,0)$ is adressed in Premoselli [16].

\section{Proof of Theorem 0.1}

By conformal invariance, letting $\tilde{g}=\varphi^{4} g$ for some smooth positive function $\varphi$, we get that $u$ solves (0.5) if and only if $\frac{u}{\varphi}$ solves

$$
\Delta_{\tilde{g}} u+\frac{1}{8} S_{\tilde{g}} u=\frac{1}{4}\left(\Lambda-\frac{1}{3} \tau^{2}\right) u^{5}+\frac{\left|\sigma_{\theta}+D W_{\theta}\right|^{2}}{8 \varphi^{12} u^{7}}+\frac{\left|E_{\theta}\right|^{2}+\left|B_{\theta}\right|^{2}}{8 \varphi^{8} u^{3}} .
$$

By the resolution of the Yamabe problem, see Aubin [1, Schoen [17] and Trüdinger [19], we can make $S_{\tilde{g}}$ constant. We separate the proof into the three cases of the theorem. The results in points (1) and (2) of Theorem 0.1 were expected from [6] and 12 . Here we provide a slightly different, and probably easier, proof.

(1) Assume first that $Y(M, g) \leq 0$. We fix $\tilde{g}$ such that $S_{\tilde{g}} \leq 0$ is constant. Integrating (1.1) with respect to $d v_{\tilde{g}}$, it is clear that $K(\Lambda, \tau)<0$ is a necessary condition for the existence of a solution to (1.1). Conversely, if we assume that $K(\Lambda, \tau)<0$ and $Y(M, g)<0$, then $u_{-} \equiv \varepsilon$ is a subsolution of (1.1) for $\varepsilon>0$ sufficiently small, while $u_{+} \equiv T$ is a supersolution of (1.1) for $T>0$ sufficiently large. By the sub and supersolution method we then get a solution $u_{-} \leq u \leq u_{+}$ of (1.1). In the case $Y(M, g)=0$, we let $s>0$, and for $\delta>0$ solve

$$
\Delta_{\tilde{g}} u_{\delta}+s u_{\delta}=\frac{\delta}{4} K+\Phi,
$$

where $K=K(\Lambda, \tau)$, and $\Phi=\left|\sigma_{\theta}+D W_{\theta}\right|^{2}+\left|E_{\theta}\right|^{2}+\left|B_{\theta}\right|^{2}$. We have that $u_{\delta} \rightarrow u_{0}$ in $L^{\infty}$ as $\delta \rightarrow 0$, where $u_{0}$ solves $\Delta_{\tilde{g}} u_{0}+s u_{0}=\Phi$. Since $\Phi \geq 0$ and $\Phi \not \equiv 0$, we get by the maximum principle that $u_{0}>0$ in $M$. Hence $u_{\delta}>0$ for $\delta>0$ small, and for $t>0$ sufficiently small it is easily checked that $u_{-}=t u_{\delta}>0$ is a subsolution of (1.1). As before, by the sub and supersolution method, we obtain a solution $u_{-} \leq u \leq u_{+}$of (1.1). This proves (1) in Theorem 0.1 .

(2) Now we assume that $Y(M, g)>0$ and that $K(\Lambda, \tau) \leq 0$. We fix $\tilde{g} \in[g]$ such that $S_{\tilde{g}}>0$ is constant. Then, given $T>0$ sufficiently large, $u_{+} \equiv T$ is a supersolution of (1.1). For $\delta>0$ we let $u_{\delta}$ solve

$$
\Delta_{\tilde{g}} u_{\delta}+\frac{1}{8} S_{\tilde{g}} u_{\delta}=\frac{\delta}{4}\left(\Lambda-\frac{1}{3} \tau^{2}\right)+\frac{\left|E_{\theta}\right|^{2}+\left|B_{\theta}\right|^{2}}{8 \varphi^{8}} .
$$


Clearly, $u_{\delta} \rightarrow u_{0}$ in $L^{\infty}$ as $\delta \rightarrow 0$, where $u_{0}$ solves

$$
\Delta_{\tilde{g}} u_{0}+\frac{1}{8} S_{\tilde{g}} u_{0}=\frac{\left|E_{\theta}\right|^{2}+\left|B_{\theta}\right|^{2}}{8 \varphi^{8}} .
$$

There holds that $u_{0}>0$ in $M$ since $(E, B) \not \equiv(0,0)$. In particular, $u_{\delta}>0$ for $\delta>0$ sufficiently small. We fix such a $\delta>0$ sufficiently small, and let $u_{\varepsilon}=\varepsilon u_{\delta}$. Then

$$
\begin{aligned}
\Delta_{\tilde{g}} u_{\varepsilon}+\frac{1}{8} S_{\tilde{g}} u_{\varepsilon} & =\varepsilon \frac{\delta}{4}\left(\Lambda-\frac{1}{3} \tau^{2}\right)+\varepsilon \frac{\left|E_{\theta}\right|^{2}+\left|B_{\theta}\right|^{2}}{8 \varphi^{8}} \\
& \leq \frac{1}{4}\left(\Lambda-\frac{1}{3} \tau^{2}\right) u_{\varepsilon}^{5}+\frac{\left|\sigma_{\theta}+D W_{\theta}\right|^{2}}{8 \varphi^{12} u_{\varepsilon}^{7}}+\frac{\left|E_{\theta}\right|^{2}+\left|B_{\theta}\right|^{2}}{8 \varphi^{8} u_{\varepsilon}^{3}}
\end{aligned}
$$

for $\varepsilon>0$ sufficiently small since, for such $\varepsilon$ 's, $u_{\varepsilon}^{5} \leq \varepsilon \delta$ and $\frac{1}{u_{\varepsilon}^{3}} \geq \varepsilon$ at any point in $M$. In particular, $u_{-} \equiv u_{\varepsilon}>0$ is a subsolution of (1.1) when $\varepsilon>0$ is sufficiently small. Then we can choose $\varepsilon>0$ such that $u_{-} \leq u_{+}$, and by the sub and supersolution method we get a solution $u_{-} \leq u \leq u_{+}$of (1.1). This proves (2) in Theorem 0.1 .

(3) At last we assume that $Y(M, g)>0$ and $K(\Lambda, \tau)>0$. The existence of $\theta_{\star}$ can be obtained from the results in Hebey, Pacard and Pollack [11. The proof in [1] relied on the mountain pass theorem. Here we provide a much simpler argument, with the price to pay being that we lose explicit control on $\theta_{\star}$ (a lower bound can be obtained from [11]). Here again we fix $\tilde{g} \in[g]$ such that $S_{\tilde{g}}>0$ is constant. We let $u_{0}>0$ be given by (1.2) and define $u_{\varepsilon}=\varepsilon u_{0}$ for $\varepsilon>0$. As above, $u_{\varepsilon}$ turns out to be a subsolution of (1.1) for $\varepsilon>0$ sufficiently small. Assume we have a solution $u_{\theta}$ of (1.1) for some $\theta>0$. Then $u_{\theta}$ is a supersolution of (1.1) for $\theta^{\prime} \leq \theta$. Choosing $\varepsilon>0$ sufficiently small such that $u_{\varepsilon} \leq u_{\theta}$ in $M$, we obtain a solution of (1.1) for all $\theta^{\prime} \leq \theta$ by the sub and supersolution method. In particular, the set $\mathcal{S}$ consisting of

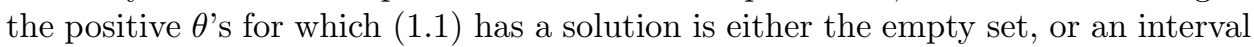
like $\left(0, \theta_{\star}\right)$, where $\theta_{\star} \leq+\infty$. Let $\varepsilon_{0}>0$ be such that

$$
\frac{1}{8} S_{\tilde{g}} \varepsilon_{0} \geq \frac{1}{4} K(\Lambda, \tau) \varepsilon_{0}^{5}+\varepsilon_{0}^{2}
$$

By the definition of $E_{\theta}, B_{\theta}, W_{\theta}, \sigma_{\theta}$, we get that $u_{+} \equiv \varepsilon_{0}$ is a supersolution of (1.1) for $\theta>0$ sufficiently small. Choosing $\varepsilon>0$ such that $u_{\varepsilon} \leq u_{+}$, we obtain a solution of (1.1) by the sub and supersolution method. In particular, there exists $\theta_{0}>0$ such that $\left(0, \theta_{0}\right) \subset \mathcal{S}$, and $\mathcal{S}$ is not empty. At this point, it remains to prove that $\theta_{\star}<+\infty$, and thus that there exists $\theta>0$ for which (1.1) does not have a solution. We follow the argument in Hebey, Pacard and Pollack [11]. Integrating (1.1) there holds that

$$
\begin{aligned}
\frac{S_{\tilde{g}}}{8} \int_{M} u d v_{\tilde{g}} & =\frac{1}{4} K(\Lambda, \tau) \int_{M} u^{5} d v_{\tilde{g}}+\theta^{2} \int_{M} \frac{\Phi_{1}}{u^{7}} d v_{\tilde{g}}+\theta^{2} \int_{M} \frac{\Phi_{2}}{u^{3}} d v_{\tilde{g}} \\
& \geq \frac{1}{4} K(\Lambda, \tau) \int_{M} u^{5} d v_{\tilde{g}}+\theta^{2} \int_{M} \frac{\Phi_{2}}{u^{3}} d v_{\tilde{g}}
\end{aligned}
$$


where $\Phi_{1}, \Phi_{2} \geq 0$ are nonnegative functions and $\Phi_{2} \not \equiv 0$. By Hölder's inequality, $\int u \leq V_{\tilde{g}}^{4 / 5}\left(\int u^{5}\right)^{1 / 5}$, where $V_{\tilde{g}}$ is the volume of $M$ with respect to $\tilde{g}$, while

$$
\begin{aligned}
\int_{M} \Phi_{2}^{5 / 12} d v_{\tilde{g}} & =\int_{M} \Phi_{2}^{5 / 12} \frac{1}{u^{5 / 4}} u^{5 / 4} d v_{\tilde{g}} \\
& \leq\left(\int_{M} \frac{\Phi_{2}}{u^{3}} d v_{\tilde{g}}\right)^{5 / 12}\left(\int_{M} u^{15 / 7} d v_{\tilde{g}}\right)^{7 / 12} \\
& \leq V_{\tilde{g}}^{1 / 3}\left(\int_{M} \frac{\Phi_{2}}{u^{3}} d v_{\tilde{g}}\right)^{5 / 12}\left(\int_{M} u^{5} d v_{\tilde{g}}\right)^{1 / 4} .
\end{aligned}
$$

Letting $X=\left(\int_{M} u^{5} d v_{\tilde{g}}\right)^{4 / 5}$, it follows from (1.3) and (1.4) that

$$
\begin{aligned}
\frac{\sqrt{K(\Lambda, \tau)}\left(\int_{M} \Phi_{2}^{5 / 12} d v_{\tilde{g}}\right)^{6 / 5} \theta}{V_{\tilde{g}}^{2 / 5}} & \leq \frac{K(\Lambda, \tau)}{4} X+\frac{\theta^{2}\left(\int_{M} \Phi_{2}^{5 / 12} d v_{\tilde{g}}\right)^{12 / 5}}{V_{\tilde{g}}^{4 / 5} X} \\
& \leq \frac{S_{\tilde{g}}}{8} V_{\tilde{g}}^{4 / 5} .
\end{aligned}
$$

As is easily checked, (1.5) is impossible when $\theta>0$ is large. In particular, (1.1) does not have a solution when $\theta>0$ is large and we get that $\theta_{\star}<+\infty$. This proves (3) in Theorem 0.1

\section{Proof of Theorem 0.2 when $K<0$}

We assume that $K(\Lambda, \tau)<0$ and let $\left(u_{\alpha}\right)_{\alpha}$ be a sequence of solutions of (0.7). Then

$$
\Delta_{g} u_{\alpha}+\frac{1}{8} S_{g} u_{\alpha}=\frac{1}{4} K_{\alpha} u_{\alpha}^{5}+\frac{\Phi_{\alpha}}{u_{\alpha}^{7}}+\frac{\Psi_{\alpha}}{u_{\alpha}^{3}}+k_{\alpha},
$$

where $K_{\alpha}=\Lambda_{\alpha}-\frac{1}{3} \tau_{\alpha}^{2}$ is such that $K_{\alpha} \rightarrow K(\Lambda, \tau)$ as $\alpha \rightarrow+\infty, \Phi_{\alpha}, \Psi_{\alpha} \geq 0$, and $\Phi_{\alpha} \rightarrow \Phi, \Psi_{\alpha} \rightarrow \Psi$ and $k_{\alpha} \rightarrow 0$ in $C^{0}$ as $\alpha \rightarrow+\infty$ for some smooth functions $\Phi, \Psi$ with $\Psi \not \equiv 0$. In the present setting,

$$
\Phi_{\alpha}=\frac{\left|\sigma_{\alpha}+D W_{\alpha}\right|^{2}}{8} \text { and } \Psi_{\alpha}=\frac{\left|E_{\alpha}\right|^{2}+\left|B_{\alpha}\right|^{2}}{8} .
$$

Let $x_{\alpha} \in M$ be such that $u_{\alpha}\left(x_{\alpha}\right)=\max _{M} u_{\alpha}$. Since $K(\Lambda, \tau)<0$, there holds that $K_{\alpha}<0$ for $\alpha \gg 1$. Then,

$$
\frac{1}{8} S_{g} u_{\alpha}\left(x_{\alpha}\right)+\frac{1}{4}\left|K_{\alpha}\right| u_{\alpha}\left(x_{\alpha}\right)^{5} \leq \frac{\Phi_{\alpha}\left(x_{\alpha}\right)}{u_{\alpha}\left(x_{\alpha}\right)^{7}}+\frac{\Psi_{\alpha}\left(x_{\alpha}\right)}{u_{\alpha}\left(x_{\alpha}\right)^{3}}+\left\|k_{\alpha}\right\|_{L^{\infty}},
$$

and we get that there exists $C>0$ such that $u_{\alpha} \leq C$ in $M$ for all $\alpha$. Given $s>0$ we let $h_{s}=\frac{1}{8} S_{g}+s$, and we fix $s \gg 1$ such that $h_{s} \geq 1$. Given $\delta>0$ we solve

$$
\left\{\begin{array}{l}
\Delta_{g} u_{\alpha, \delta}+h_{s} u_{\alpha, \delta}=\Phi_{\alpha}+\Psi_{\alpha}+\frac{\delta}{4} K_{\alpha} \\
\Delta_{g} u_{\delta}+h_{s} u_{\delta}=\Phi+\Psi+\frac{\delta}{4} K \\
\Delta_{g} r_{\alpha}+h_{s} r_{\alpha}=k_{\alpha}
\end{array}\right.
$$

where $K=K(\Lambda, \tau)$. Then $u_{\alpha, \delta} \rightarrow u_{\delta}$ in $L^{\infty}$ as $\alpha \rightarrow+\infty$, while $u_{\delta} \rightarrow u_{0}$ in $L^{\infty}$ as $\delta \rightarrow 0$, where

$$
\Delta_{g} u_{0}+h_{s} u_{0}=\Phi+\Psi .
$$

Since $\Phi, \Psi \geq 0$ and $\Psi \not \equiv 0$, there holds that $u_{0}>0$ in $M$. In particular, $u_{\delta}>0$ in $M$ for $\delta>0$ sufficiently small, and fixing $\delta>0$ small, we get that there exists 
$\varepsilon_{0}>0$ such that $u_{\alpha, \delta} \geq \varepsilon_{0}$ for all $\alpha \gg 1$. Now we define $\varphi_{\alpha}=t u_{\alpha, \delta}+r_{\alpha}$ for $t>0$, where $r_{\alpha}$ is as in (2.3). There holds that $r_{\alpha} \rightarrow 0$ in $L^{\infty}$ as $\alpha \rightarrow+\infty$. Given $t>0$ sufficiently small we have that

$$
\begin{aligned}
\Delta_{g} \varphi_{\alpha}+h_{s} \varphi_{\alpha} & =t \Phi_{\alpha}+t \Psi_{\alpha}+\frac{\delta}{4} t K_{\alpha}+k_{\alpha} \\
& \leq \frac{\Phi_{\alpha}}{\varphi_{\alpha}^{7}}+\frac{\Psi_{\alpha}}{\varphi_{\alpha}^{3}}+\frac{1}{4} K_{\alpha} \varphi_{\alpha}^{5}+k_{\alpha}
\end{aligned}
$$

for all $\alpha \gg 1$, since $K_{\alpha}<0$ for $\alpha \gg 1, u_{\alpha, \delta} \rightarrow u_{\delta}$ in $L^{\infty}$ as $\alpha \rightarrow+\infty$, and $u_{\alpha, \delta} \geq \varepsilon_{0}>0$ and $r_{\alpha} \rightarrow 0$ in $L^{\infty}$. We fix $t>0$ sufficiently small. Then, for $\alpha \gg 1$,

$$
\begin{aligned}
& \Delta_{g}\left(u_{\alpha}-\varphi_{\alpha}\right)+h_{s}\left(u_{\alpha}-\varphi_{\alpha}\right) \\
& \geq \frac{1}{4} K_{\alpha}\left(u_{\alpha}^{5}-\varphi_{\alpha}^{5}\right)+\Phi_{\alpha}\left(u_{\alpha}^{-7}-\varphi_{\alpha}^{-7}\right)+\Psi_{\alpha}\left(u_{\alpha}^{-3}-\varphi_{\alpha}^{-3}\right) \\
& \geq 0
\end{aligned}
$$

at any point such that $u_{\alpha} \leq \varphi_{\alpha}$. Hence, $\varphi_{\alpha} \leq u_{\alpha}$ in $M$ for all $\alpha \gg 1$ by the maximum principle. Since $\varphi_{\alpha} \geq \frac{1}{2} t \varepsilon_{0}$ for $\alpha \gg 1$, we get that there exists $C>1$ such that

$$
\frac{1}{C} \leq u_{\alpha} \leq C
$$

in $M$ for all $\alpha$. By (2.1) and elliptic theory we then get that the $u_{\alpha}$ 's are bounded in $C^{1, \eta}$ for $0<\eta<1$. In particular, up to a subsequence, $u_{\alpha} \rightarrow u$ in $C^{1, \eta^{\prime}}$ for $\eta^{\prime} \in(0, \eta)$ and some smooth positive solution $u$ of (0.1). This proves the stability of $(0.1)$.

\section{Proof of Theorem 0.2 when $K=0$}

First we prove stability. We assume that $K(\Lambda, \tau)=0$ and let $\left(u_{\alpha}\right)_{\alpha}$ be a bounded sequence in $H^{1}$ of solutions of (0.7). Then

$$
\Delta_{g} u_{\alpha}+\frac{1}{8} S_{g} u_{\alpha}=\frac{1}{4} K_{\alpha} u_{\alpha}^{5}+\frac{\Phi_{\alpha}}{u_{\alpha}^{7}}+\frac{\Psi_{\alpha}}{u_{\alpha}^{3}}+k_{\alpha},
$$

where $K_{\alpha}=\Lambda_{\alpha}-\frac{1}{3} \tau_{\alpha}^{2}$ is such that $K_{\alpha} \rightarrow 0$ as $\alpha \rightarrow+\infty, \Phi_{\alpha}, \Psi_{\alpha} \geq 0$, and $\Phi_{\alpha} \rightarrow \Phi$, $\Psi_{\alpha} \rightarrow \Psi$ and $k_{\alpha} \rightarrow 0$ in $C^{0}$ as $\alpha \rightarrow+\infty$ for some smooth functions $\Phi, \Psi$ with $\Psi \not \equiv 0$. Namely, $\Phi_{\alpha}$ and $\Psi_{\alpha}$ are given by (2.2). Moreover we have here that $\left\|u_{\alpha}\right\|_{H^{1}} \leq C$ for some $C>0$ and all $\alpha$. Replacing $K_{\alpha}$ by $\tilde{K}_{\alpha}=\min \left(0, K_{\alpha}\right)$ in (2.3), we get as above that there exists $\varepsilon_{0}>0$ such that $u_{\alpha} \geq \varepsilon_{0}$ in $M$ for all $\alpha$. Now we let $x_{\alpha} \in M$ be such that $u_{\alpha}\left(x_{\alpha}\right)=\max _{M} u_{\alpha}$. By contradiction we assume that $u_{\alpha}\left(x_{\alpha}\right) \rightarrow+\infty$ as $\alpha \rightarrow+\infty$. Let $\mu_{\alpha}>0$ be given by $\mu_{\alpha}^{-1 / 2}=u_{\alpha}\left(x_{\alpha}\right)$, and let $\tilde{u}_{\alpha}$ be given by

$$
\tilde{u}_{\alpha}(x)=\mu_{\alpha}^{\frac{1}{2}} u_{\alpha}\left(\exp _{x_{\alpha}}\left(\mu_{\alpha} x\right)\right)
$$

for $x \in \mathbb{R}^{3}$. Also let $\tilde{g}_{\alpha}=\left(\exp _{x_{\alpha}}^{\star} g\right)\left(\mu_{\alpha} x\right)$. There holds that

$$
\Delta_{\tilde{g}_{\alpha}} \tilde{u}_{\alpha}+\mu_{\alpha}^{2} h_{\alpha} \tilde{u}_{\alpha}=K_{\alpha} \tilde{u}_{\alpha}^{5}+\mu_{\alpha}^{5 / 2} \tilde{R}_{\alpha},
$$

where

$$
h_{\alpha}(x)=\frac{1}{8} S_{g}\left(\exp _{x_{\alpha}}\left(\mu_{\alpha} x\right)\right), \tilde{R}_{\alpha}(x)=R_{\alpha}\left(\exp _{x_{\alpha}}\left(\mu_{\alpha} x\right)\right),
$$

and $R_{\alpha}$ is some function such that $\left\|R_{\alpha}\right\|_{L^{\infty}} \leq C$ for some $C>0$ and all $\alpha$. By construction, $0 \leq \tilde{u}_{\alpha} \leq 1$. By elliptic theory and (3.2) we then get that there exists $\tilde{u}: \mathbb{R}^{3} \rightarrow \mathbb{R}, \tilde{u} \in \dot{H}^{1}$, such that $\tilde{u}_{\alpha} \rightarrow \tilde{u}$ in $C_{l o c}^{2}\left(\mathbb{R}^{3}\right)$. Then, $0 \leq \tilde{u} \leq 1, \tilde{u}(0)=1$, and 
by (3.2), since $K_{\alpha} \rightarrow 0$ and $\mu_{\alpha} \rightarrow 0$, we get that $\tilde{u}$ is harmonic - a contradiction with Liouville's theorem. This implies that there exists $C>0$ such that $u_{\alpha} \leq C$ for all $\alpha$. We conclude to the stability of (0.1) as in the preceding section.

Concerning compactness, we let $\left(u_{\alpha}\right)_{\alpha}$ be any sequence of solutions of (0.1). By Theorem 0.1 this implies that $Y(M, g)>0$ (since if not the case, the equation does not have any solution). As above, when discussing stability we obtain that there exists $\varepsilon_{0}>0$ such that $u_{\alpha} \geq \varepsilon_{0}$ in $M$ for all $\alpha$. The proof can even be made much simpler. We just need to solve

$$
\Delta_{g} u_{0}+h_{s} u_{0}=\Phi+\Psi .
$$

Then we can note that $u_{0}>0$, that

$$
\Delta_{g} \varphi+h_{s} \varphi \leq \frac{\Phi}{\varphi^{7}}+\frac{\Psi}{\varphi^{3}}
$$

for $\varphi=t u_{\delta}$ when $0<t \ll 1$, and that

$$
\Delta_{g}\left(u_{\alpha}-\varphi\right)+h_{s}\left(u_{\alpha}-\varphi\right) \geq 0
$$

at any point where $u_{\alpha} \leq \varphi$. We conclude as in the preceding section the existence of $\varepsilon_{0}$. Now we let $\tilde{g} \in[g]$ be such that $S_{\tilde{g}}>0$ is constant, and let $\tilde{u}_{\alpha}=\frac{u_{\alpha}}{\varphi}$. Then $\tilde{u}_{\alpha}$ solves

$$
\Delta_{\tilde{g}} \tilde{u}_{\alpha}+\frac{1}{8} S_{\tilde{g}} \tilde{u}_{\alpha}=\frac{|\sigma+D W|^{2}}{8 \varphi^{12} \tilde{u}_{\alpha}^{7}}+\frac{|E|^{2}+|B|^{2}}{8 \varphi^{8} \tilde{u}_{\alpha}^{3}} .
$$

If $x_{\alpha}$ is a point where $\tilde{u}_{\alpha}$ is maximum, since $\Delta_{\tilde{g}} \tilde{u}_{\alpha}\left(x_{\alpha}\right) \geq 0$ and $S_{\tilde{g}}>0$, we get that $\tilde{u}_{\alpha}\left(x_{\alpha}\right) \leq C$ for some $C>0$ and all $\alpha$. In particular, there exists $C>0$ such that $u_{\alpha} \leq C$ for all $\alpha$. This, together with the lower bound $u_{\alpha} \geq \varepsilon_{0}$, implies the compactness of (0.1) thanks to standard elliptic theory.

As a final remark on the $K=0$ case, suppose $Y(M, g) \leq 0$. By Theorem 0.1 . there exists $u_{\alpha}>0$ which solves (0.1) for any $K_{\alpha}<0$. Let $\left(K_{\alpha}\right)_{\alpha}$ be such that $K_{\alpha}<0$ for all $\alpha$ and $K_{\alpha} \rightarrow 0$ as $\alpha \rightarrow+\infty$. Choosing $\tilde{g} \in[g]$ such that $S_{\tilde{g}} \leq 0$, and up to changing $u_{\alpha}$ into $\tilde{u}_{\alpha}=\varphi^{-1} u_{\alpha}$ for some smooth positive function $\varphi$, there holds that

$$
\Delta_{\tilde{g}} \tilde{u}_{\alpha}+\frac{1}{8} S_{\tilde{g}} \tilde{u}_{\alpha}=\frac{1}{4} K_{\alpha} \tilde{u}_{\alpha}^{5}+\frac{|\sigma+D W|^{2}}{8 \varphi \tilde{u}_{\alpha}^{7}}+\frac{|E|^{2}+|B|^{2}}{8 \varphi \tilde{u}_{\alpha}^{3}}
$$

for all $\alpha$. Integrating (3.4) we see that, necessarily, $\int_{M} \tilde{u}_{\alpha}^{5} d v_{g} \rightarrow+\infty$ as $\alpha \rightarrow+\infty$. In particular, (0.1) when $K=0$ possesses perturbations which themselves possess sequences of solutions with unbounded energy. This provides another illustration, in addition to the toy model mentioned in the introduction, of the fact that (0.1) when $K=0$ cannot be bounded and stable.

\section{Proof of Theorem 0.2 when $K>0$}

We assume here that $K>0$ and follow the more involved analysis in Druet and Hebey [8]. We let $\left(u_{\alpha}\right)_{\alpha}$ be a sequence of solutions of (0.7). Then

$$
\Delta_{g} u_{\alpha}+\frac{1}{8} S_{g} u_{\alpha}=\frac{1}{4} K_{\alpha} u_{\alpha}^{5}+\frac{\Phi_{\alpha}}{u_{\alpha}^{7}}+\frac{\Psi_{\alpha}}{u_{\alpha}^{3}}+k_{\alpha},
$$

where $K_{\alpha}=\Lambda_{\alpha}-\frac{1}{3} \tau_{\alpha}^{2}$ is such that $K_{\alpha} \rightarrow K$ as $\alpha \rightarrow+\infty, K>0, \Phi_{\alpha}, \Psi_{\alpha} \geq 0$, and $\Phi_{\alpha} \rightarrow \Phi, \Psi_{\alpha} \rightarrow \Psi$ and $k_{\alpha} \rightarrow 0$ in $C^{0}$ as $\alpha \rightarrow+\infty$ for some smooth functions $\Phi, \Psi$ with $\Psi \not \equiv 0$. Namely, $\Phi_{\alpha}$ and $\Psi_{\alpha}$ are given by (2.2). We separate the proof into 
several lemmas. Following the argument in Section 2 we first prove that the $u_{\alpha}$ 's are bounded from below far from 0 .

Lemma 4.1. Let $\left(u_{\alpha}\right)_{\alpha}$ solves (4.1). There exists $\varepsilon_{0}>0$ such that $u_{\alpha} \geq \varepsilon_{0}$ for all $\alpha$.

Proof of Lemma 4.1. Given $s>0$ we let $h_{s}=\frac{1}{8} S_{g}+s$, and we fix $\theta \gg 1$ such that $h_{s} \geq 1$. Given $\delta>0$ we solve

$$
\left\{\begin{array}{l}
\Delta_{g} u_{\alpha, \delta}+h_{s} u_{\alpha, \delta}=\Phi_{\alpha}+\Psi_{\alpha}, \\
\Delta_{g} u_{\delta}+h_{s} u_{\delta}=\Phi+\Psi \\
\Delta_{g} r_{\alpha}+h_{s} r_{\alpha}=k_{\alpha} .
\end{array}\right.
$$

Then $u_{\alpha, \delta} \rightarrow u_{\delta}$ in $L^{\infty}$ as $\alpha \rightarrow+\infty$, while $u_{\delta} \rightarrow u_{0}$ in $L^{\infty}$ as $\delta \rightarrow 0$, where

$$
\Delta_{g} u_{0}+h_{s} u_{0}=\Phi+\Psi \text {. }
$$

Since $\Phi, \Psi \geq 0$ and $\Psi \not \equiv 0$, there holds that $u_{0}>0$ in $M$. In particular, $u_{\delta}>0$ in $M$ for $\delta>0$ sufficiently small, and fixing $\delta>0$ small, we get that there exists $\varepsilon_{0}>0$ such that $u_{\alpha, \delta} \geq \varepsilon_{0}$ for all $\alpha \gg 1$. Now we define

$$
\varphi_{\alpha}=t u_{\alpha, \delta}+r_{\alpha}
$$

for $t>0$, where $r_{\alpha}$ is as in (4.2). There holds that $r_{\alpha} \rightarrow 0$ in $L^{\infty}$ as $\alpha \rightarrow+\infty$. Given $t>0$ sufficiently small we have that

$$
\Delta_{g} \varphi_{\alpha}+h_{s} \varphi_{\alpha} \leq \frac{\Phi_{\alpha}}{\varphi_{\alpha}^{7}}+\frac{\Psi_{\alpha}}{\varphi_{\alpha}^{3}}+k_{\alpha}
$$

for all $\alpha \gg 1$. We fix $t>0$ sufficiently small. Then, for $\alpha \gg 1$,

$$
\begin{aligned}
& \Delta_{g}\left(u_{\alpha}-\varphi_{\alpha}\right)+h_{s}\left(u_{\alpha}-\varphi_{\alpha}\right) \\
& \geq \frac{1}{4} K_{\alpha} u_{\alpha}^{5}+\Phi_{\alpha}\left(u_{\alpha}^{-7}-\varphi_{\alpha}^{-7}\right)+\Psi_{\alpha}\left(u_{\alpha}^{-3}-\varphi_{\alpha}^{-3}\right) \\
& \geq 0
\end{aligned}
$$

at any point such that $u_{\alpha} \leq \varphi_{\alpha}$. Hence, $\varphi_{\alpha} \leq u_{\alpha}$ in $M$ for all $\alpha \gg 1$ by the maximum principle. Since $\varphi_{\alpha} \geq \frac{1}{2} t \varepsilon_{0}$ for $\alpha \gg 1$, we get a lower bound for the $u_{\alpha}$ 's, and this ends the proof of the lemma.

Thanks to Lemma 4.1, there holds that $\left|\Delta_{g} u_{\alpha}\right| \leq C u_{\alpha}^{5}$ in $M$ for all $\alpha$. We assume in what follows that the $u_{\alpha}$ 's blow up. By elliptic theory, the sole $C^{0}$-norm of the $u_{\alpha}$ 's is involved. In other words, we assume that

$$
\lim _{\alpha \rightarrow+\infty}\left\|u_{\alpha}\right\|_{L^{\infty}}=+\infty \text {. }
$$

Then, as is by now classical in blow-up theory, there exists $C>0$ such that for any $\alpha$, there exist $N_{\alpha} \in \mathbb{N}^{\star}$ and a set $\mathcal{S}_{\alpha}$ of $N_{\alpha}$ critical points of $u_{\alpha}$, denoted by

$$
\mathcal{S}_{\alpha}=\left\{\left(x_{1, \alpha}, x_{2, \alpha}, \ldots, x_{N_{\alpha}, \alpha}\right)\right\},
$$

such that

$$
d_{g}\left(x_{i, \alpha}, x_{j, \alpha}\right)^{\frac{1}{2}} u_{\alpha}\left(x_{i, \alpha}\right) \geq 1
$$

for all $i, j \in\left\{1, \ldots, N_{\alpha}\right\}, i \neq j$, and

$$
\left(\min _{i=1, \ldots, N_{\alpha}} d_{g}\left(x_{i, \alpha}, x\right)\right)^{\frac{1}{2}} u_{\alpha}(x) \leq C
$$


for all $x \in M$ and all $\alpha$. Now we let $\left(x_{\alpha}\right)_{\alpha}$ be a sequence of points in $M$ and $\left(\rho_{\alpha}\right)_{\alpha}$ be a sequence of positive real numbers with $0<\rho_{\alpha}<\frac{1}{7} i_{g}(M)$ such that

$$
\begin{aligned}
& x_{\alpha} \text { is a critical point of } u_{\alpha}, \text { and } \\
& d_{g}\left(x_{\alpha}, x\right)^{\frac{1}{2}} u_{\alpha}(x) \leq C \text { for all } x \in B_{x_{\alpha}}\left(7 \rho_{\alpha}\right)
\end{aligned}
$$

for all $\alpha$. By (4.4) and (4.5), we get that (4.6) holds true for $x_{\alpha}=x_{i_{\alpha}, \alpha}$, where $\left(i_{\alpha}\right)_{\alpha}$ is a sequence of integers in $\left\{1, \ldots, N_{\alpha}\right\}$ up to taking $\rho_{\alpha}$ sufficiently small, and in particular less than $1 / 8^{t h}$ of the minimum distance between the $x_{i, \alpha}$ 's and $x_{\alpha}$ for $i \neq i_{\alpha}$. In addition to (4.6) we consider the assumption that

$$
\rho_{\alpha}^{\frac{1}{2}} \sup _{B_{x_{\alpha}}\left(6 \rho_{\alpha}\right)} u_{\alpha} \rightarrow+\infty
$$

as $\alpha \rightarrow+\infty$. The rescaling arguments, as in Claim 1 of Druet [7], then give that, up to a subsequence, $x_{\alpha} \rightarrow x_{0}$ as $\alpha \rightarrow+\infty$ for some $x_{0} \in M$, and

$$
\mu_{\alpha}^{\frac{1}{2}} u_{\alpha}\left(\exp _{x_{\alpha}}\left(\mu_{\alpha} x\right)\right) \rightarrow \bar{u}(x)
$$

in $C_{l o c}^{1}\left(\mathbb{R}^{3}\right)$ as $\alpha \rightarrow+\infty$, where $\mu_{\alpha}>0$ is given by $u_{\alpha}\left(x_{\alpha}\right)=\mu_{\alpha}^{-1 / 2}$, and

$$
\bar{u}(x)=\left(\frac{1}{1+\frac{K|x|^{2}}{12}}\right)^{1 / 2} .
$$

In particular, there holds that $\frac{\rho_{\alpha}}{\mu_{\alpha}} \rightarrow+\infty$ as $\alpha \rightarrow+\infty$, and $\mu_{\alpha} \rightarrow 0$ as $\alpha \rightarrow+\infty$. Now we define $\varphi_{\alpha}:\left(0, \rho_{\alpha}\right) \mapsto \mathbb{R}^{+}$by

$$
\varphi_{\alpha}(r)=\frac{1}{\left|\partial B_{x_{\alpha}}(r)\right|_{g}} \int_{\partial B_{x_{\alpha}}(r)} u_{\alpha} d \sigma_{g},
$$

where $\left|\partial B_{x_{\alpha}}(r)\right|_{g}$ is the volume of $\partial B_{x_{\alpha}}(r)$ for the metric induced by $g$. By (4.8),

$$
\left(\mu_{\alpha} r\right)^{\frac{1}{2}} \varphi_{\alpha}\left(\mu_{\alpha} r\right) \rightarrow r^{\frac{1}{2}}\left(1+\frac{K r^{2}}{12}\right)^{-\frac{1}{2}}
$$

in $C_{l o c}^{1}([0,+\infty))$ as $\alpha \rightarrow+\infty$. We define $r_{\alpha} \in\left(2 R_{0} \mu_{\alpha}, \rho_{\alpha}\right]$ by

$$
\begin{aligned}
r_{\alpha}=\sup \{r \in( & \left.2 R_{0} \mu_{\alpha}, \rho_{\alpha}\right) \text { s.t. } \\
& \left.s^{\frac{1}{2}} \varphi_{\alpha}(s) \text { is nonincreasing in }\left(2 R_{0} \mu_{\alpha}, r\right)\right\},
\end{aligned}
$$

where $R_{0}^{2}=\frac{12}{K}$ and $\varphi_{\alpha}$ is as in (4.9). We know thanks to (4.10) that

$$
\frac{r_{\alpha}}{\mu_{\alpha}} \rightarrow+\infty
$$

as $\alpha \rightarrow+\infty$. Also we have that

$$
\begin{aligned}
& r^{\frac{1}{2}} \varphi_{\alpha} \text { is nonincreasing in }\left(2 R_{0} \mu_{\alpha}, r_{\alpha}\right), \\
& \text { and that }\left(r^{\frac{1}{2}} \varphi_{\alpha}(r)\right)^{\prime}\left(r_{\alpha}\right)=0 \text { if } r_{\alpha}<\rho_{\alpha} .
\end{aligned}
$$

Now we prove that the following sharp pointwise estimates on the $u_{\alpha}$ 's hold true. 
Lemma 4.2. Let $\left(x_{\alpha}\right)_{\alpha}$ and $\left(\rho_{\alpha}\right)_{\alpha}$ be such that (4.6) and (4.7) hold true. Up to passing to a subsequence, $\rho_{\alpha} \rightarrow 0, \rho_{\alpha}^{1 / 2} u_{\alpha}\left(x_{\alpha}\right) \rightarrow+\infty$ and

$$
u_{\alpha}\left(x_{\alpha}\right) \rho_{\alpha} u_{\alpha}\left(\exp _{x_{\alpha}}\left(\rho_{\alpha} x\right)\right) \rightarrow \frac{\lambda}{|x|}+H(x)
$$

in $C_{\text {loc }}^{2}\left(B_{0}(5) \backslash\{0\}\right)$ as $\alpha \rightarrow+\infty$ for some $\lambda>0$ and some harmonic function $H$ in $B_{0}(5)$ which satisfies $H(0)=0$.

Proof of Lemma 4.2, Mimicking the analysis in Druet and Hebey [8] we get that there exists $C>0$ such that

$$
u_{\alpha}(x)+d_{g}\left(x_{\alpha}, x\right)\left|\nabla u_{\alpha}(x)\right| \leq C \mu_{\alpha}^{\frac{1}{2}} d_{g}\left(x_{\alpha}, x\right)^{-1}
$$

for all $x \in B_{x_{\alpha}}\left(6 r_{\alpha}\right) \backslash\left\{x_{\alpha}\right\}$. As a consequence, $r_{\alpha}^{2}=O\left(\mu_{\alpha}\right)$, where $\mu_{\alpha}$ is as in (4.8). First we prove that, after passing to a subsequence,

$$
\mu_{\alpha}^{-\frac{1}{2}} r_{\alpha} u_{\alpha}\left(\exp _{x_{\alpha}}\left(r_{\alpha} x\right)\right) \rightarrow \frac{R_{0}}{|x|}+H(x)
$$

in $C_{l o c}^{2}\left(B_{0}(5) \backslash\{0\}\right)$ as $\alpha \rightarrow+\infty$ where $H$ is some harmonic function in $B_{0}(5)$. Also, we prove that if $r_{\alpha}<\rho_{\alpha}$, then we have that $H(0)=R_{0}$. We set, for $x \in \mathbb{R}^{3}$,

$$
\hat{u}_{\alpha}(x)=\mu_{\alpha}^{-\frac{1}{2}} r_{\alpha} u_{\alpha}\left(\exp _{x_{\alpha}}\left(r_{\alpha} x\right)\right) \text { and } \hat{g}_{\alpha}(x)=\left(\exp _{x_{\alpha}}^{\star} g\right)\left(r_{\alpha} x\right) \text {. }
$$

We know that $\hat{g}_{\alpha} \rightarrow \xi$ in $C_{\text {loc }}^{2}\left(\mathbb{R}^{3}\right)$ since $r_{\alpha} \rightarrow 0$ as $\alpha \rightarrow+\infty$. We also know thanks to (4.14) that $\hat{u}_{\alpha}(x) \leq C|x|^{-1}$ in $B_{0}(6) \backslash\{0\}$. Using (4.1) we then get that $\Delta_{\hat{g}_{\alpha}} \hat{u}_{\alpha}=\hat{F}_{\alpha}$ in $B_{0}(6)$, where

$$
\left|\hat{F}_{\alpha}(x)\right| \leq C \frac{\mu_{\alpha}^{2}}{r_{\alpha}^{2}}|x|^{-5}
$$

in $B_{0}(6) \backslash\{0\}$ thanks to Lemma 4.1. By (4.12) and standard elliptic theory we easily get that $\hat{u}_{\alpha} \rightarrow \hat{U}$ in $C_{l o c}^{1}\left(B_{0}(5) \backslash\{0\}\right)$ as $\alpha \rightarrow+\infty$, where $\Delta_{\xi} \hat{U}=0$ in $B_{0}(5) \backslash\{0\}$. Moreover, we have that $0 \leq \hat{U}(x) \leq C_{4}|x|^{-1}$. We can write that

$$
\hat{U}(x)=\frac{\lambda}{|x|^{n-2}}+H(x)
$$

for some $\lambda \geq 0$ and some function $H$ harmonic in $B_{0}(5)$. In order to get (4.15) it remains to prove that $\lambda=R_{0}$. This can be done by integrating the equation $\Delta_{\hat{g}_{\alpha}} \hat{u}_{\alpha}=\hat{F}_{\alpha}$ satisfied by $\hat{u}_{\alpha}$ on $B_{0}(1)$. Indeed, integrating the equation we get that

$$
-\int_{\partial B_{0}(1)} \partial_{\nu} \hat{u}_{\alpha} d \sigma_{\hat{g}_{\alpha}}=\int_{B_{0}(1)} \hat{F}_{\alpha} d v_{\hat{g}_{\alpha}} .
$$

Thanks to (4.8) and (4.14) we easily obtain that

$$
\int_{B_{0}(1)} \hat{F}_{\alpha} d v_{\hat{g}_{\alpha}} \rightarrow \frac{K}{4} \int_{\mathbb{R}^{3}}\left(1+\frac{|x|^{2}}{R_{0}^{2}}\right)^{-\frac{5}{2}} d x
$$

as $\alpha \rightarrow+\infty$. It is sufficient to remark that

$$
\frac{K}{4} \int_{\mathbb{R}^{3}}\left(1+\frac{|x|^{2}}{R_{0}^{2}}\right)^{-\frac{5}{2}} d x=\omega_{2} R_{0}
$$

and that

$$
-\int_{\partial B_{0}(1)} \partial_{\nu} \hat{u}_{\alpha} d \sigma_{\hat{g}_{\alpha}} \rightarrow-\lambda \omega_{2}
$$


as $\alpha \rightarrow+\infty$ to conclude that $\lambda=R_{0}$. In the case $r_{\alpha}<\rho_{\alpha}$, we can use (4.13) to get that $\left(r^{1 / 2} \varphi(r)\right)^{\prime}(1)=0$, where

$$
\varphi(r)=\frac{1}{\omega_{2} r^{2}} \int_{\partial B_{0}(r)} \hat{U} d \sigma=\frac{R_{0}}{r}+H(0) .
$$

We conclude that $H(0)=R_{0}$ if $r_{\alpha}<\rho_{\alpha}$. In particular, in order to end the proof of Lemma 4.2, it remains to prove that $H(0)=0$. For this we apply the Pohozaev identity in Druet and Hebey [8]. Let $X_{\alpha}$ be the vector field whose coordinates in the exponential chart at $x_{\alpha}$ are $X_{\alpha}^{i}=x^{i}$. Applying the identity in $\Omega_{\alpha}=B_{x_{\alpha}}\left(r_{\alpha}\right)$, we get that

$$
\begin{aligned}
& \int_{\Omega_{\alpha}}\left(\nabla u_{\alpha}\left(X_{\alpha}\right)+\frac{1}{2} u_{\alpha}\right) \Delta_{g} u_{\alpha} d v_{g} \\
& =O\left(\int_{\Omega_{\alpha}} d_{g}\left(x_{\alpha}, x\right)^{2}\left|\nabla u_{\alpha}\right|^{2} d v_{g}\right) \\
& +\int_{\partial \Omega_{\alpha}}\left(\frac{1}{2}\left(X_{\alpha}, \nu\right)_{g}\left|\nabla u_{\alpha}\right|^{2}-\nabla u_{\alpha}\left(X_{\alpha}\right) \partial_{\nu} u_{\alpha}-\frac{1}{2} u_{\alpha} \partial_{\nu} u_{\alpha}\right) d \sigma_{g},
\end{aligned}
$$

where $\nu$ is the unit outer normal of $\partial \Omega_{\alpha}$. By (4.15),

$$
\begin{aligned}
& \int_{\partial \Omega_{\alpha}}\left(\frac{1}{2}\left(X_{\alpha}, \nu\right)_{g}\left|\nabla u_{\alpha}\right|^{2}-\nabla u_{\alpha}\left(X_{\alpha}\right) \partial_{\nu} u_{\alpha}-\frac{1}{2} u_{\alpha} \partial_{\nu} u_{\alpha}\right) d \sigma_{g} \\
& =\left(\frac{1}{2} \omega_{2} R_{0} H(0)+o(1)\right) \mu_{\alpha} r_{\alpha}^{-1} .
\end{aligned}
$$

Since $r_{\alpha}^{2}=O\left(\mu_{\alpha}\right)$ we can write that $\mu_{\alpha} r_{\alpha}=o\left(\mu_{\alpha} r_{\alpha}^{-1}\right)$. Then, by Lemma 4.1, (4.8) and (4.14), and thanks to the equation satisfied by the $u_{\alpha}$ 's, there holds that

$$
\begin{aligned}
& \int_{\Omega_{\alpha}} d_{g}\left(x_{\alpha}, x\right)^{2}\left|\nabla u_{\alpha}\right|^{2} d v_{g}=o\left(\mu_{\alpha} r_{\alpha}^{-1}\right) \text { and } \\
& \int_{\Omega_{\alpha}}\left(\nabla u_{\alpha}\left(X_{\alpha}\right)+\frac{1}{2} u_{\alpha}\right) \Delta_{g} u_{\alpha} d v_{g}=o\left(\mu_{\alpha} r_{\alpha}^{-1}\right) .
\end{aligned}
$$

Plugging (4.17) and (4.18) into (4.16) we get that $H(0)=0$. This ends the proof of Lemma 4.2.

Now we return to the situation at the beginning of the section and let

$$
d_{\alpha}=\min _{1 \leq i<j \leq N_{\alpha}} d_{g}\left(x_{i, \alpha}, x_{j, \alpha}\right),
$$

where $N_{\alpha}$ and the $x_{i, \alpha}$ 's are as in (4.4) and (4.5). By Lemma 4.2 we may assume $N_{\alpha} \geq 2$. Up to reordering the points, we may also assume that

$$
d_{\alpha}=d_{g}\left(x_{1, \alpha}, x_{2, \alpha}\right) \leq d_{g}\left(x_{1, \alpha}, x_{3, \alpha}\right) \leq \cdots \leq d_{g}\left(x_{1, \alpha}, x_{N_{\alpha}, \alpha}\right) .
$$

Thanks to Lemma 4.2 we can now end the proof of Theorem 0.2 .

Proof of Theorem 0.2 . We prove that both $d_{\alpha} \not \rightarrow 0$ and that $d_{\alpha} \rightarrow 0$. First we claim that there necessarily holds that $d_{\alpha} \nrightarrow \rightarrow 0$ and thus that, up to a subsequence, $d_{\alpha} \geq d$ for some $d>0$. In order to prove the existence of $d$ we proceed by contradiction and assume that $d_{\alpha} \rightarrow 0$ as $\alpha \rightarrow+\infty$. Let $\delta \in\left(0, \frac{1}{2} i_{g}\right)$ be given. For $x \in B_{0}\left(\delta d_{\alpha}^{-1}\right)$, we let

$$
\tilde{u}_{\alpha}(x)=d_{\alpha}^{1 / 2} u_{\alpha}\left(\exp _{x_{1, \alpha}}\left(d_{\alpha} x\right)\right)
$$


Also let $\tilde{g}_{\alpha}(x)=\left(\exp _{x_{1, \alpha}}^{\star} g\right)\left(d_{\alpha} x\right)$. Then

$$
\Delta_{\tilde{g}_{\alpha}} \tilde{u}_{\alpha}+d_{\alpha}^{2} h_{\alpha} \tilde{u}_{\alpha}=\frac{1}{4} K_{\alpha} \tilde{u}_{\alpha}^{5}+d_{\alpha}^{5 / 2} \tilde{R}_{\alpha}
$$

where $h_{\alpha}(x)=\frac{1}{8} S_{g}\left(\exp _{x_{\alpha}}\left(d_{\alpha} x\right)\right), \tilde{R}_{\alpha}(x)=R_{\alpha}\left(\exp _{x_{\alpha}}\left(d_{\alpha} x\right)\right)$, and $\left\|R_{\alpha}\right\|_{L^{\infty}} \leq C$ for some $C>0$. We clearly have that $\tilde{g}_{\alpha} \rightarrow \xi$ in $C_{l o c}^{2}\left(\mathbb{R}^{3}\right)$ as $\alpha \rightarrow+\infty$. Given $R>0$ we let $1 \leq N_{R, \alpha} \leq N_{\alpha}$ be such that $d_{g}\left(x_{1, \alpha}, x_{i, \alpha}\right) \leq R d_{\alpha}$ for all $1 \leq i \leq N_{R, \alpha}$, and $d_{g}\left(x_{1, \alpha}, x_{i, \alpha}\right)>R d_{\alpha}$ for all $N_{R, \alpha}+1 \leq i \leq N_{\alpha}$. We have that $N_{R, \alpha} \geq 2$ for all $R>1$, and $\left(N_{R, \alpha}\right)_{\alpha}$ is uniformly bounded for all $R>0$. Mimicking the arguments in Druet and Hebey [8] (see also Druet, Hebey and Vétois [10]), given $R>0$, there holds that

$$
\begin{aligned}
& \text { either } \tilde{u}_{\alpha}\left(\tilde{x}_{i, \alpha}\right)=O(1) \text { for all } 1 \leq i \leq N_{R, \alpha} \\
& \text { or } \tilde{u}_{\alpha}\left(\tilde{x}_{i, \alpha}\right) \rightarrow+\infty \text { as } \alpha \rightarrow+\infty \text { for all } 1 \leq i \leq N_{R, \alpha},
\end{aligned}
$$

where the $\tilde{u}_{\alpha}$ 's are as in (4.21), and

$$
\tilde{x}_{i, \alpha}=\frac{1}{d_{\alpha}} \exp _{x_{1, \alpha}}^{-1}\left(x_{i, \alpha}\right) .
$$

We now split the proof into the study of two cases. In the first case we assume that there exist $R>0$ and $1 \leq i \leq N_{R, \alpha}$ such that $\tilde{u}_{\alpha}\left(\tilde{x}_{i, \alpha}\right)=O(1)$. Then, by (4.23), $\tilde{u}_{\alpha}\left(\tilde{x}_{i, \alpha}\right)=O(1)$ for all $1 \leq i \leq N_{R, \alpha}$ and all $R>0$. Noting that the two equations in (4.6) are satisfied by $x_{\alpha}=x_{i, \alpha}$ and $\rho_{\alpha}=\frac{1}{8} d_{\alpha}$, it follows from (4.8) that the sequence $\left(\tilde{u}_{\alpha}\right)_{\alpha}$ is uniformly bounded in the balls $B_{\tilde{x}_{i, \alpha}}(1 / 2)$. Thus, by (4.22) and elliptic theory, the sequence $\left(\tilde{u}_{\alpha}\right)_{\alpha}$ is bounded in $C_{l o c}^{1}\left(\mathbb{R}^{3}\right)$. Up to a subsequence, still thanks to (4.22), we get that the $\tilde{u}_{\alpha}$ 's converge in $C_{l o c}^{1}\left(\mathbb{R}^{3}\right)$ as $\alpha \rightarrow+\infty$ to some $\tilde{u}$, which satisfies

$$
\Delta \tilde{u}=\frac{K}{4} \tilde{u}^{5}
$$

in $\mathbb{R}^{3}$. Moreover, $\tilde{u}$ has two critical points which are 0 and the limit $\tilde{x}_{2} \in S^{2}$ as $\alpha \rightarrow+\infty$ of the $\tilde{x}_{2, \alpha}$ 's in (4.24). By the classification result of Caffarelli, Gidas, and Spruck 4, this is impossible. In particular, we are left with the second case of our study, where we assume that there exist $R>0$ and $1 \leq i \leq N_{R, \alpha}$ such that $\tilde{u}_{\alpha}\left(\tilde{x}_{i, \alpha}\right) \rightarrow+\infty$ as $\alpha \rightarrow+\infty$. Then, by (4.23), $\tilde{u}_{\alpha}\left(\tilde{x}_{i, \alpha}\right) \rightarrow+\infty$ as $\alpha \rightarrow+\infty$ for all $1 \leq i \leq N_{R, \alpha}$ and all $R>0$. The assumptions (4.6) and (4.7) are satisfied by $x_{\alpha}=x_{1, \alpha}$ and $\rho_{\alpha}=\frac{1}{8} d_{\alpha}$. Let $\tilde{v}_{\alpha}=\tilde{u}_{\alpha}(0) \tilde{u}_{\alpha}$. By Lemma 4.1 and (4.22),

$$
\Delta_{\tilde{g}_{\alpha}} \tilde{v}_{\alpha}+d_{\alpha}^{2} h_{\alpha} \tilde{v}_{\alpha}=\frac{F_{\alpha}}{\tilde{u}_{\alpha}(0)^{4}} \tilde{v}_{\alpha}^{5}
$$

where $\left\|F_{\alpha}\right\|_{L^{\infty}} \leq C$. Noting that $\tilde{u}_{\alpha}(0) \rightarrow+\infty$ as $\alpha \rightarrow+\infty$, again mimicking arguments from Druet and Hebey [8, and Druet, Hebey and Vétois [10, we obtain with (4.25) that, up to a subsequence, $\tilde{u}_{\alpha}(0) \tilde{u}_{\alpha} \rightarrow \tilde{G}$ in $C_{l o c}^{1}\left(\mathbb{R}^{3} \backslash\left\{\tilde{x}_{i}\right\}_{i \in I}\right)$ as $\alpha \rightarrow$ $+\infty$, where the $\tilde{x}_{i}$ 's are the limits of the $\tilde{x}_{i, \alpha}$ 's in (4.24), where the index set $I$ is given by $I=\left\{1, \ldots, \lim _{R \rightarrow+\infty} \lim _{\alpha \rightarrow+\infty} N_{R, \alpha}\right\}$, and where, for any $R>0$,

$$
\begin{aligned}
\tilde{G}(x) & =\sum_{i=1}^{\tilde{N}_{R}} \frac{\Lambda_{i}}{\left|x-\tilde{x}_{i}\right|}+\tilde{H}_{R}(x) \\
& =\frac{\Lambda_{1}}{|x|}+\left(\sum_{i=2}^{\tilde{N}_{R}} \frac{\Lambda_{i}}{\left|x-\tilde{x}_{i}\right|}+\tilde{H}_{R}(x)\right)
\end{aligned}
$$


in $B_{0}(R)$, where $\Lambda_{i}>0$ for all $i, \tilde{H}_{R}$ is harmonic in $B_{0}(R), 2 \leq \tilde{N}_{R} \leq N_{2 R}$ is such that $\left|\hat{x}_{\tilde{N}_{R}}\right| \leq R$ and $\left|\hat{x}_{\tilde{N}_{R}+1}\right|>R$, and $N_{2 R, \alpha} \rightarrow N_{2 R}$ as $\alpha \rightarrow+\infty$. By Lemma 4.2. and (4.26), we get that

$$
\sum_{i=2}^{\tilde{N}_{R}} \frac{\Lambda_{i}}{\left|\tilde{x}_{i}\right|}+\tilde{H}_{R}(0)=0 .
$$

Independently, by the maximum principle, since $\tilde{G} \geq 0$ and $\left|\tilde{x}_{2}\right|=1$, there holds that

$$
\sum_{i=2}^{\tilde{N}_{R}} \frac{\Lambda_{i}}{\left|\tilde{x}_{i}\right|}+\tilde{H}_{R}(0) \geq \Lambda_{2}-\frac{\Lambda_{1}}{R}-\frac{\Lambda_{2}}{R-1} .
$$

Choosing $R \gg 1$ sufficiently large, we get a contradiction by combining (4.27) and (4.28). In particular, this proves that $d_{\alpha} \not \rightarrow 0$ as $\alpha \rightarrow+\infty$ and thus that there exists $d>0$, small, such that

$$
d_{g}\left(x_{i, \alpha}, x_{j, \alpha}\right) \geq d
$$

for all $1 \leq i<j \leq N_{\alpha}$ and all $\alpha$. Then $\left(N_{\alpha}\right)_{\alpha}$ is bounded and it can be assumed to be a constant equal to $N$ after passing to a subsequence. Let $x_{\alpha}$ be a point where $u_{\alpha}$ is maximum. By (4.3), $u_{\alpha}\left(x_{\alpha}\right) \rightarrow+\infty$ as $\alpha \rightarrow+\infty$. Up to passing to another subsequence, we then get that there exists $i \in\{1, \ldots, N\}$ such that $d_{g}\left(x_{i, \alpha}, x_{\alpha}\right) \rightarrow 0$ as $\alpha \rightarrow+\infty$ and we get that (4.6) holds true with $\rho_{\alpha}=\frac{1}{16} d$. Also we have that (4.7) holds true. Hence, by Lemma 4.2 $\rho_{\alpha} \rightarrow 0$ as $\alpha \rightarrow+\infty$ and we get a contradiction with $d>0$. The theorem is proved.

\section{References}

[1] Aubin, T., Équations différentielles non linéaires et problème de Yamabe concernant la courbure scalaire, J. Math. Pures Appl., 55, 269-296, 1976. MR0431287 (55:4288)

[2] Bartnik, R., Isenberg, J., The constraints equations. In: The Einstein equations and the large scale behavior of gravitational fields, edited by P.T.Chrusciel, H.Friedreich, Basel: Birkhäuser, 1-39, 2004. MR2098911 (2005f:83001)

[3] Beig, R., Chrusciel, P.T., and Schoen, R., KIDs are non-generic, Ann. Inst. H. Poincaré. Anal. Non Linéaire, 6, 155-194, 2005. MR2121280 (2005m:83013)

[4] Caffarelli, L. A., Gidas, B., Spruck, J., Asymptotic symmetry and local behavior of semilinear elliptic equations with critical Sobolev growth. Comm. Pure Appl. Math., 42, 271-297, 1989. MR.982351 (90c:35075)

[5] Choquet-Bruhat, Y., Isenberg, J., Pollack, D., The constraints equations for the Einsteinscalar field system on compact manifolds. Class. Quantum Grav., 24, 809-828, 2007. MR2297268 (2008a:83012)

[6] Chrusciel, P., Galloway, G., and Pollack, D., Mathematical general relativity: a sampler, Bull. Amer. Math. Soc. (N.S.), 47, 567-638, 2010. MR2721040 (2011j:53152)

[7] Druet, O., Compactness for Yamabe metrics in low dimensions, Internat. Math. Res. Notices, 23, 1143-1191, 2004. MR2041549 (2005b:53056)

[8] Druet, O., and Hebey, E., Stability and instability for Einstein-scalar field Lichnerowicz equations on compact Riemannian manifolds, Math. Z., 263, 33-67, 2009. MR2529487 (2010h:58028)

[9] , Stability for strongly coupled critical elliptic systems in a fully inhomogeneous medium, Analysis and PDEs, 2, 305-359, 2009. MR2603801 (2011k:58032)

[10] Druet, O., Hebey, E., and Vétois, J., Bounded stability for strongly coupled critical elliptic systems below the geometric threshold of the conformal Laplacian, J. Funct. Anal., 258, 999-1059, 2010. MR2558186 (2011a:53053) 
[11] Hebey, E., Pacard, F., Pollack, D., A variational analysis of Einstein-scalar field Lichnerowicz equations on compact Riemannian manifolds. Comm. Math. Phys., 278, 117-132, 2008. MR2367200 (2009c:58041)

[12] Isenberg, J., Constant mean curvature solutions of the Einstein constraint equations on closed manifolds, Class. Quantum Grav., 12, 2249-2274, 1995. MR1353772 (97a:83013)

[13] Isenberg, J., Maxwell, D., Pollack, D., A gluing construction for non-vacuum solutions of the Einstein-constraint equations, Adv. Theor. Math. Phys., 9, 129-172, 2005. MR2193370 (2006j:83004)

[14] Maxwell, D., Rough solutions of the Einstein constraint equations on compact manifolds, J. Hyp. Diff. Eq., 2, 521-546, 2005. MR2151120 (2006d:58027)

[15] _ A class of solutions of the vacuum Einstein constraint equations with freely specified mean curvature, Math. Res. Lett., 16, 627-645, 2009. MR2525029 (2010j:53057)

[16] Premoselli, B., Equations de contraintes en relativité générale, Preprint, 2011.

[17] Schoen, R.M., Conformal deformation of a Riemannian metric to constant scalar curvature, J. Differential Geometry, 20, 479-495, 1984. MR788292(86i:58137)

[18] _ On the number of constant scalar curvature metrics in a conformal class, Differential Geometry: A Symposium in Honor of Manfredo do Carmo, Proc. Int. Conf. (Rio de Janeiro, 1988). Pitman Monogr. Surveys Pure Appl. Math., vol. 52, Longman Sci. Tech., Harlow, 311-320, 1991. MR1173050(94e:53035)

[19] Trudinger, N.S., Remarks concerning the conformal deformation of Riemannian structures on compact manifolds, Ann. Scuola Norm. Sup. Pisa, 22, 265-274, 1968. MR0240748 (39:2093)

Département de Mathématiques, Université de Cergy-Pontoise, Site de Saint-Martin,

2 avenue Adolphe Chauvin, 95302 Cergy-Pontoise cedex, France

E-mail address: Emmanuel.Hebey@math.u-cergy.fr

Département de Mathématiques, Université de Cergy-Pontoise, Site de Saint-Martin,

2 avenue Adolphe Chauvin, 95302 Cergy-Pontoise cedex, France

E-mail address: giona.veronelli@math.u-cergy.fr

Current address: Département de Mathématiques, Institut Galilée, Université Paris 13,

99 avenue Jean-Baptiste Clément, 93430 Villetaneuse, France

E-mail address: veronelli@math.univ-paris13.fr 